\title{
Stoichiometric Analysis of the Weight-Decrease in the Enzymatic Treatment of Cotton Fiber: A Guide to Real-Time Monitoring System for the Treatment
}

\author{
Shigeyuki Nakano*1, Ryüi Hirase*1, Kazuhiko Komurasaki*1, \\ Ahmed EL Salmawy ${ }^{*}$ and Yoshiharu Kimura ${ }^{\star 2}$. \\ ${ }^{*}$ Hyogo Prefectural Institute of Industrial Research, Technicäl Center for Textiles \\ 1790- 496 Nomura-cho Nishiwaki, Hyogo 677-0054, Japan \\ *2 Department of Polymer Science and Engineering, Kyoto Institute of Technology \\ Matsugasaki, Kyoto 606-8585, Japan
}

\begin{abstract}
Enzymatic hydrolysis of cotton fiber was studied in detail to find out a good way to monitor the weight loss of fiber precisely. In this enzymatic treatment the linear relationship between the weight loss and the glucose formation was confirmed. Stoichiometric analysis of the hydrolysis products revealed that both glucose and oligosaccharides are formed from cellobiose in a constant ratio to give the linearity. This may be attributed to the cellobiose hydrase and polymerase activities involved in the cellulase used (originated from Filamentous fungt). Based on this finding, a new monitoring system using the chemi-luminescence of luminol was examined for assessing the glucose formation, which was related with the total weight loss of cotton fiber. This method is superior to other methods in terms of shortness of measuring time and applicable for monitoring the weight loss of dyed fabrics.
\end{abstract}

\section{Introduction}

It has generally been known that the appearance and handling of fabrics is improved by the denier-decreasing treatment in which the fibers are allowed to lose its volume in knitted or woven state. [1,2] Enzymatic hydrolysis is an ordinary tool for the denier-decreasing treatment of cotton fiber, because cellulose can be hydrolyzed by the action of cellulase in a fast rate. This hydrolysis of cotton, however, has not easily been controlled thus far, because no good monitoring device has been available. In the practical enzymatic treatment, the factory engineers have relied on their experience for controlling the degree of hydrolysis of cotton fabrics. Therefore, development of a simple monitoring system for assessing the weight decrease of cotton fiber has been requested.

Since cellulose can be enzymatically hydrolyzed into cellobiose (Fig.1), analysis of cellobiose is to be the best way for assessing the degree of hydrolysis. However, the ordinary cellulases commercially available involve several enzymes such as cellobiose hydrase by which the produced cellobiose is hydrolyzed into glucose or reversibly condensed to higher oligo-saccharides. In fact, HPLC analysis of the hydrolysis products of cotton fiber supported the formation of both glucose and cellobiose. In 1996, S. V. Chikkodi reported that the amount of glucose formed in this enzymatic hydrolysis can be
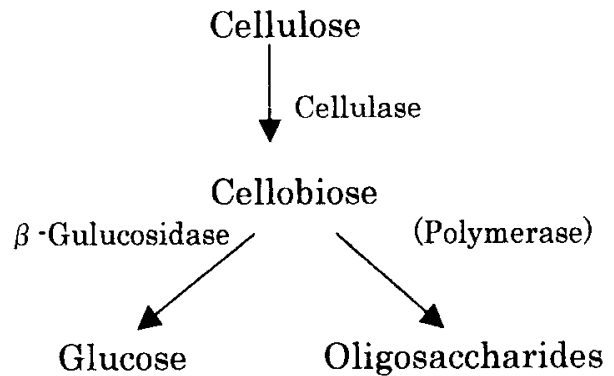

Fig. 1 Reaction paths in the enzymatic hydrolysis of cellulose. 
linearly correlated with the total weight loss of cotton fiber[3], although its reason has never been known until now. This occasional correlation should be very convenient to assess the degree of hydrolysis or weight loss of cotton fiber during the enzymatic hydrolysis. So, we tried to analyze why this linear relationship is established and then examined to use a simple chemi-luminescence method for real-time measurement of the amount of glucose produced in situ. In this method, the produced glucose was reacted with a glucose oxidase to generate a stoichiometric amount of hydrogen peroxide that was quantitatively analyzed by the photo-luminescence induced by the peroxidase-mediated luminol reaction $[4,5]$. This method is so simple and sensitive as to allow detection of a low concentration of glucose formed in the early stage of the treatment.

\section{Experimental}

\subsection{Materials}

American cotton made in Sun Forking Valley with an average diameter of $13.0 \mu \mathrm{m}$ and an average length of $23.5 \mathrm{~mm}$ was used. It was bleached in an aqueous solution of nonionic surfactant Noigen 2NT, Daiich Kougyou Seiyaku Co. Ltd (Osaka) $(1.0 \mathrm{~g} / \mathrm{l})$ and sodium carbonate $(2.0 \mathrm{~g} / \mathrm{l})$ at $90{ }^{\circ} \mathrm{C}$ for $40 \mathrm{~min}$, rinsed twice in hot water and twice in water, and finally dried at $105{ }^{\circ} \mathrm{C}$ for $2 \mathrm{~h}$.

A cellulase originated from Filamentous fungi was supplied by Nagase Sangyo Co. Ltd (Osaka). It was a mixture of various types of cellulases. Its activity was $846 \mathrm{U} / \mathrm{g}$ protein as measured [6] where $1 \mathrm{U}$ corresponded to the enzymatic activity to produce $1 \mu \mathrm{mol}$ of glucose from an excess $(125 \mathrm{~g})$ of carboxymethyl cellulose (CMC) at $40^{\circ} \mathrm{C}$ for 1 min. Luminol was purchased from Kishida Chemical Co. Ltd (Osaka). Glucose oxidase (GOD) originated from Asperigillus niger and Horseradish peroxidase (POD) were purchased from Sigma-Aldrich Japan K.K. (Tokyo). A luminol reagent used for chemiluminescence was prepared by mixing luminol $(15 \mathrm{mg} / \mathrm{dl})$, glucose oxidase $(25 \mathrm{mg} / \mathrm{dl})$, and peroxidase $(5 \mathrm{mg} / \mathrm{dl})$ in phosphate buffered solution (PBS; $\mathrm{pH}=6$ ).

\subsection{Method of enzymatic treatment}

Cotton fiber $(3 \mathrm{~g})$ was dipped in $300 \mathrm{ml}$ of a cellulase solution $(1.0 \mathrm{~g} / \mathrm{l})$ in a buffer $(\mathrm{pH}=5)$ consisting of $0.5 \mathrm{~g} / \mathrm{l}$ sodium acetate and $0.2 \mathrm{~g} / \mathrm{l}$ acetic acid at $45{ }^{\circ} \mathrm{C}$. After a prescribed time the fiber was taken out from the solution, washed thoroughly with hot water $\left(80^{\circ} \mathrm{C}\right)$ for 20 min, filtered, and dried at $105{ }^{\circ} \mathrm{C}$ for $2 \mathrm{~h}$.

\subsection{HPLC measurements}

HPLC analysis was conducted on a system of Hitachi L-7000 series with dual pumps. Both Buffer A $\left(6.2 \mathrm{~g} / \mathrm{l}-\mathrm{H}_{3} \mathrm{BO}_{3}\right.$ and $0.36 \mathrm{~g} / \mathrm{l}$ $\mathrm{NaOH})$ and Buffer $\mathrm{B}\left(18.6 \mathrm{~g} / \mathrm{l}-\mathrm{H}_{3} \mathrm{BO}_{3}\right.$ and 6.0 $\mathrm{g} / \mathrm{l}-\mathrm{NaOH}$ ) were flowed in gradient at $70{ }^{\circ} \mathrm{C}$ at a flowing rate of $0.4 \mathrm{ml} / \mathrm{min}$. The gradient program was as follows: $0.0-3.5 \mathrm{~min}(100 \% \mathrm{~A})$, 3.5-13.5 min (a gradient from 100\% A to $100 \%$ B), $13.5-24.5 \mathrm{~min} .(100 \% \mathrm{~B})$, and $24.6-40 \mathrm{~min}$ $(100 \% \mathrm{~A})$. The eluent was guided into a tube reactor $(0.33 \mathrm{~mm} \phi)$ maintained at $150{ }^{\circ} \mathrm{C}$ and mixed with a phosphoric acidphenyhydrazine solution (a mixture of $6.0 \mathrm{ml}$ of phosphoric acid) that was injected at 0.5 $\mathrm{ml} / \mathrm{min}$. The reaction time was $1.13 \mathrm{~min}$. The reacted eluent was then analyzed with a fluorescence detector (Hitachi Co. Ltd.) at $365 \mathrm{~nm}$ and $470 \mathrm{~nm}$ in excitation and fluorescence wave lengths, respectively. A typical chromatogram showed two peaks at 6.3 and $27.8 \mathrm{~min}$ in elution time which were attributed to cellobiose and glucose, respectively. Their amounts were estimated from the peak areas in reference to the calibration curve prepared.

\subsection{Estimation of glucose by luminol reaction}

Fig.2 shows the reaction scheme for the detection of glucose by luminol reaction in which two types of enzymes are involved. The glucose oxidase catalyzes the stoichiometric formation of hydrogen peroxide, while the peroxidase catalyzes the oxidation of such diacylhydrazides as luminol by hydrogen peroxide. The photons, generated in this light emitting reaction of luminol, can be counted as the scintillation counting of the radioactive rays by using a Lumicounter $2500 \mathrm{~S}$ photocounter (Microtech Nichion Co. Ltd. (Chiba)).

A $5 \mathrm{ml}$ amount was sampled from the enzyme-treating solution and added with $5 \mathrm{ml}$ of the luminol reagent. The light-emitting solution was installed with the photocounter to collect the photocounts for 10 sec in an 


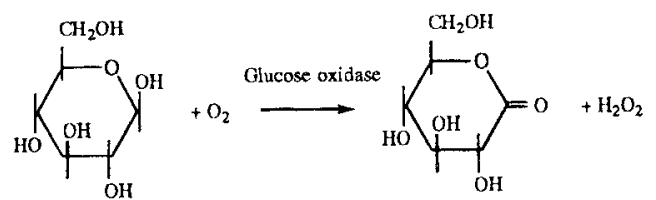<smiles>Nc1cccc(C(=O)[O-])c1C(=O)[O-]</smiles>

Fig.2 Luminol reaction for chemiluminescence

interval of $30 \mathrm{sec}$. The count collection was repeated until the collected counts reached a constant. In average, $5 \mathrm{~min}$ was necessary for one measurement.

\section{Results and Discussion}

3.1 Analysis of the hydrolysis products of cotton

Fig.3 shows a typical time-dependent formation of glucose and cellobiose during the cellulase treatment of the cotton fiber as measured by HPLC. The production of cellobiose was very fast in the initial stage of treatment and slowly increased thereafter. The formation of glucose, in return, increased steadily with treatment time. This result supported the fact that the enzymatic hydrolysis of cotton fiber gives both glucose and cellobiose.

In the actual enzymatic treatment of cotton fabric the weight decrease has usually been judged by the treatment time. However, the hydrolysis reaction is strongly affected by the slight change in reaction condition, particularly, by the change in enzyme activity which likely happens with different lot of the enzyme and with different work-up condition. Fig. 4 shows the weight decrease of the cotton fiber with treatment time that was observed for five runs. It is known that the weight decrease fluctuated in large degree. This result manifests how difficult is to control the weight decrease only by the treating time in the practical enzymatic treatment.

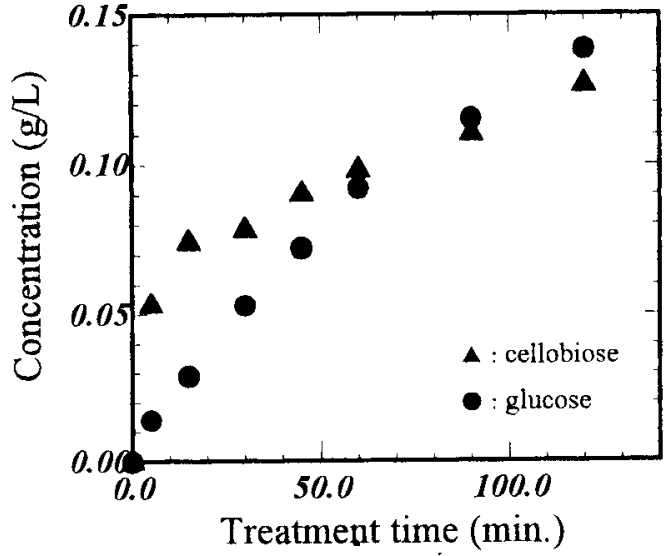

Fig.3 Changes in concentration of glucose and cellobiose with treatment time.

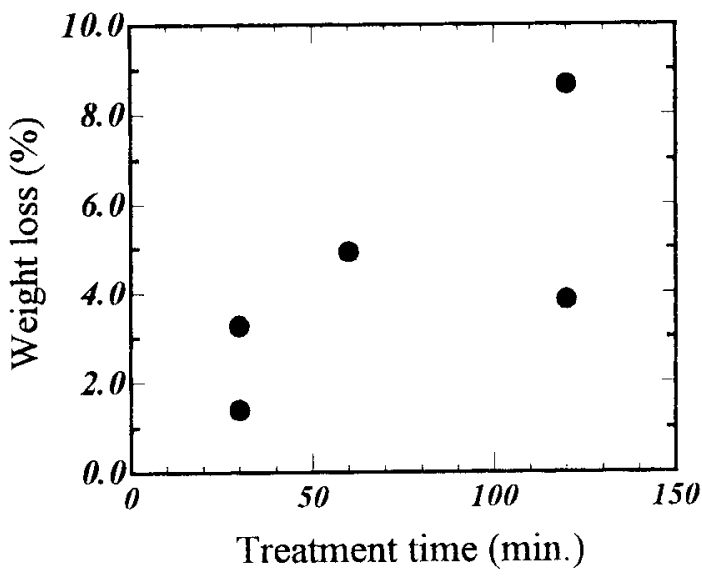

Fig.4 Weight decreases of the cotton fiber with treatment time.

As Chikkodi reported before, [3] the weight loss of cotton fabric is roughly proportional to the amount of glucose produced in the enzymatic treatment of cotton fabric. Fig.5 shows the corresponding relation for which the data of Fig.4 are used and plotted as a function of the amount of glucose produced. A clearly linear relationship is shown between the weight loss and the amount of glucose released.

\subsection{Meaning of the liner relation}

The liner relation between the weight decrease and the amount of glucose released is somewhat surprising, because the insoluble cellulose is hydrolyzed by lipase in a heterogeneous system for which the kinetic analysis is almost impossible. Therefore, we 
tried to analyze this relation from a viewpoint of mechanistic or stoichiometric feature. As shown in Fig.1, the enzymatic hydrolysis of cellulose is assumed to produce glucose and cellobiose in addition to other products. With this assumption, the following stoichiometric relation is obtained:

$$
W=W_{1}+W_{2}+W_{3}
$$

where $W$ denotes the weight decrease of cotton fiber, and $W_{1}, W_{2}$, and $W_{3}$ denote the amounts of glucose, cellobiose, and other products released by the enzymatic hydrolysis. For the liner relationship to stand between the weight loss and the amount of glucose, the following relation is assumed:

$$
\mathrm{W}=\mathrm{kW}_{\mathrm{l}}
$$

where $\mathrm{k}$ is a constant. Combination of Eq. (1) and Eq.(2) gives an idea that $W_{1}$ is also proportional to $\mathrm{W}_{2}$ and $\mathrm{W}_{3}$. Fig.6(a) shows the plot of $W_{2}$ as a function of $W_{1}$ in the actual treatment. 'The plot does not pass through the origin of the coordinate because of the fast rate of production of cellobiose in the very beginning of the treatment. However, the plot becomes linear with respect to $W_{1}$ with a gentle slope because the increase of cellobiose $\left(\mathrm{W}_{2}\right)$ became steady as shown in Fig.3. Similarly, the plot of $W_{3}$ as to $W_{1}$ is also linear without passing through the origin (Fig.6(b)). Since the $W_{2}-W_{1}$ and $W_{3}-W_{1}$ plots have the positive and negative $y$-intercepts, respectively, a better liner relationship is shown between $\mathrm{W}_{2}+\mathrm{W}_{3}$ and $\mathrm{W}_{1}$, and the deviation in the initial stage is much smaller (Fig.6(c)). As shown in Fig.1, cellobiose is thought to be the primary hydrolysis product which is subsequently converted to glucose by the hydrolysis and to higher oligosaccharides by the condensation. $\mathrm{W}_{2}$ is more responsible for the large initial weight loss than $W_{3}$, whereas the both contributions of $\mathrm{W}_{2}$ and $\mathrm{W}_{3}$ are constant in the later stage.

From the slopes of the linear plots of $\left(\mathrm{W}_{2}+\mathrm{W}_{3}\right) \cdot \mathrm{W}_{1}$ and $\mathrm{W} \cdot \mathrm{W}_{1}$, the following empirical equations can be deduced:

$$
\begin{aligned}
& \mathrm{W}_{2}+\mathrm{W}_{3}=4.2 \mathrm{~W}_{1} \\
& \mathrm{~W}=5.2 \mathrm{~W}_{1}
\end{aligned}
$$

These equations suggest that the production of glucose is only $20 \%$ of the weight loss of the cotton fiber and that $75 \%$ is retained in the enzyme reaction system as unknown products probably oligosaccharides as postulated above. Because cotton fiber contains many kinds of

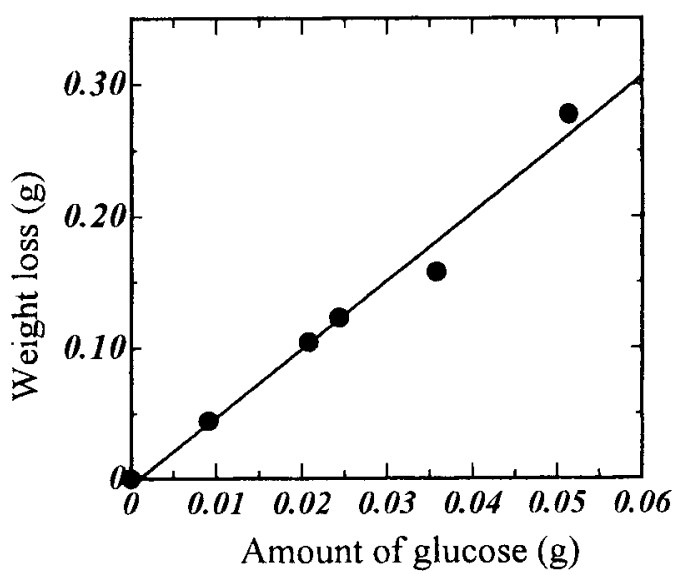

Fig.5 Relationship between the weight loss And the amount of glucose released.

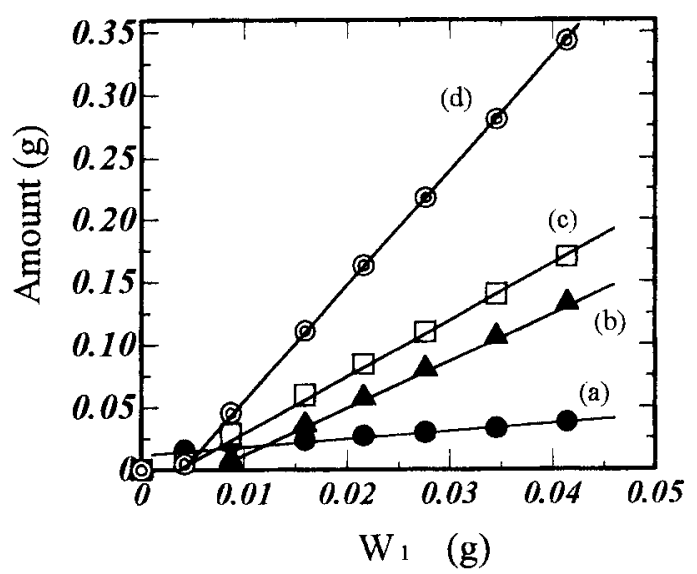

Fig.6 Plots of $W_{2}(a), W_{3}(B)$ and $W_{2}+W_{3}(c)$ compared with the plot of $W$ against $W_{1}(d)$

contaminants other than cellulose, their removal with the cellulose hydrolysis may contribute to the total weight loss. Also, the naps present in the fiber surface may drop off without hydrolysis to lead a relatively large weight loss. These contributions are all included in $W_{3}$, which is occasionally proportional to $\mathrm{W}_{1}$.

In another possible interpretation, both the hydrolysis to glucose and the polymerization to oligosaccharides from cellobiose competitively proceed in a constant ratio. The observation of the constant coefficient of $\left(\mathrm{W}_{2}+\mathrm{W}_{3}\right) / \mathrm{W}_{1}=4.2$ may be a strong support for this interpretation. If the cellulase involves the activities of both cellobiose hydrase and polymerase in a certain 


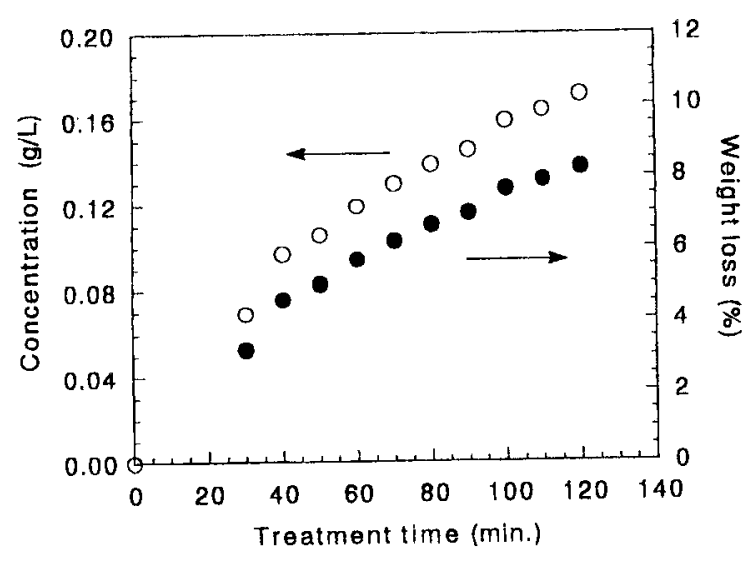

Fig.7 Changes in glucose concentration(O) determined by the chemiluminescence method and weight loss (O)during the enzymatic treatment.

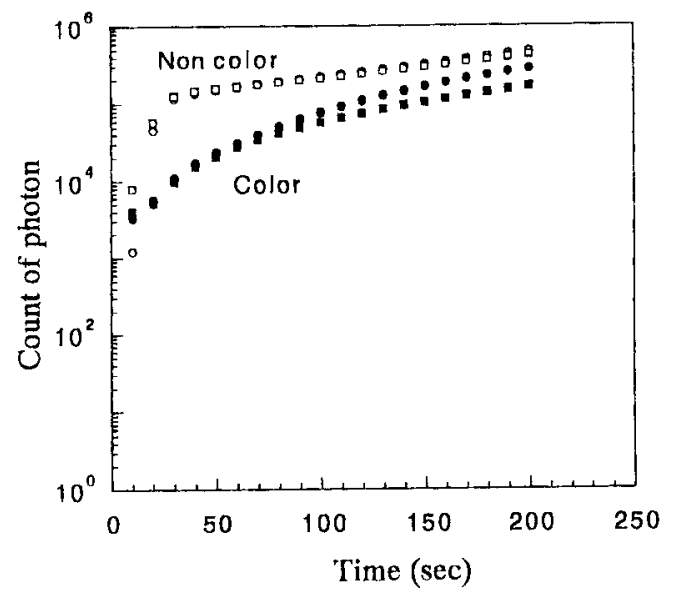

Fig. 8 Changes in photon counts by chemi-

Luminescence with the measurement time in the presence ( $\boldsymbol{\square}$ ) and absence ( $\square$ ) of dyestuff.

ratio, the glucose formation may be correlated with the oligosacchride formation and finally with the total weight loss of fiber.

The linear relationship between $\mathrm{W}$ and $\mathrm{W}_{1}$ ought to be valid in any types of cellulosic fibers, although the coefficient will slightly differ depending on the hydrolyzability and morphology of cotton fiber in conjunction with the enzyme accessibility to the fiber surface.

3.3 Chemiluminescence method for

measuring glucose amount.

The chemiluminescence method shown in Experimental has often been utilized for detection of glucose in biochemistry field. $[4,5]$
The method involves glucose oxidasemediated oxidation of glucose and peroxidasemediated luminol reaction (Fig.2). The photons generated by this reaction were counted and integrated to relate the concentration of glucose in situ. Fig. 7 shows a typical time-dependent increase in glucose amount during the enzymatic treatment as determined by the chemiluminescence method. The curve is very close to that of the glucose increase shown in Fig.3, supporting the applicability of this method.

Since this method is very simple and sensitive, the glucose amount can be monitored in real-time of the treatment. This is the most favorable feature of this method [7] in its application in factory. The aforementioned HPLC method needs a long elusion time (ca. $20 \mathrm{~min}$ ) to fail with real-time analysis of glucose. The alternative fluorescence or absorption method is not either so sensitive to detect such a low concentration of glucose.

We also examined the error range of the chemiluminescence measurement when the dyed cotton was treated and the dyestuff was eluted from the fiber into the enzyme solution. As a model reaction of it, a red disperse dyestuff (Kayalon Polyester Red BR-S, Nippon Kayaku Co. Ltd. (Tokyo)) was put into the enzyme solution in a concentration of 0.14 wt\% prior to the treatment of cotton fiber. Fig. 8 shows the changes in photon counts by chemiluminescence with the measurement time in the presence and absence of the dyestaff. The difference in photon counts between the two cases was very large in the early stage (below $150 \mathrm{sec}$ ). When the measurement time was over $200 \mathrm{sec}$, however, the photon counts measured in the presence of dyestaff reached a level almost identical to those measured in the absence of dyestaff (the difference was $35 \%$ at largest.) Therefore, the glucose amount can be measured with small error even for the dyed fabrics. The initial deviation of the photon counts in the early stage of the measurement may be due to the reaction of the intermediately formed hydrogen peroxide with the dyestaff, which is competitive to its reaction with luminol. These facts suggest a real superiority of the present method. 


\section{Conclusion}

The linear relationship between the weight loss and the glucose formation was re-confirmed in the enzymatic treatment of cotton fiber. It was found out that both glucose and oligosaccharides are formed in a constant ratio to give the linearity. Based on this linear relationship, the chemi-luminescence method using the luminol reaction was examined for monitoring glucose formation from which the total weight loss of cotton fiber was estimated precisely. This method is applicabile for monitoring the weight loss of dyed fabrics.

\section{Reference}

1. O. Tanida, Sen'i Gakkaishi, 50(2),75 (1994)

2. M. Kawano, K. Mitsuishi, T. Takeuchi, Sen'T Gakkaishi, 51(1), 44 (1995)

3. S. V. Chikkodi, Text. Chem. Color, 28(3), 28 (1996)

4. M. Yamada, S. Suzuki, Fundamentals of Instrumental Analysis. 4, 38 (1987)

5.F.Inaba,T.Gotou, M.Nakano, "Luminescence no Sokutei to Oyo" NTS Co. Ltd ('Tokyo), p65 (1999)

6. Nihon Kagaku-kai,"Bunseki Kagaku Jikken Handbook" Maruzenn (Tokyo), p580 (1987)

7. M. Ouki, T. Toshiaki, M. Tanaka, H. Chihara, "Encyclopedic Dictionary of Chemistry" Tokyo-Kagaku-dojin (Tokyo), p1732 (1989) 